\title{
Efecto del confinamiento COVID-19 sobre la composición corporal en residentes con discapacidad intelectual
}

\author{
Effect of COVID-19 lockdown on body composition of residents with \\ intelectual disability
}

\begin{abstract}
Resumen
El propósito de este estudio ha sido comprobar el efecto del confinamiento COVID-19 durante 17 semanas sobre el grado actividad física y la composición corporal de las personas residentes con discapacidad intelectual. Se ha utilizado un diseño intragrupo de medidas repetidas pre y post confinamiento. Han participado 42 personas adultas con diferentes grados de discapacidad intelectual. Para evaluar el grado de actividad física se ha utilizado el Global Physical Activity Questionnaire (GPAQ) y para el análisis de composición corporal la bioimpedancia eléctrica (InBody-230). Los resultados indican que durante el confinamiento ha existido una reducción media del $89 \%$ en todos los valores de actividad física y un incremento del $28 \%$ de los hábitos sedentarios $(\mathrm{p}<0.001)$. Se ha producido una pérdida generalizada de masa muscular $(2.5 \% ; p<0.01)$, lo que podría interpretarse como una sarcopenia temporal forzada. Como conclusión, se pone de manifiesto la importancia de los programas de actividad física en este colectivo.
\end{abstract}

Palabras clave

Actividad física, cuestionario GPAQ, hábitos sedentarios, bioimpedancia.

\begin{abstract}
The purpose of this study was to determinate the effect of COVID-19 lockdown measures after 17 weeks on the physical activity level and body composition of residents with intellectual disabilities. An intra-group repeated measures design was used before and after lockdown. The study involved 42 adults with different degrees of intellectual disability. The Global Physical Activity Questionnaire (GPAQ) was used to evaluate the degree of physical activity and the electrical bioimpedance (InBody-230) for the analysis of body composition. The results obtained reveal that during lockdown there was an average reduction of $89 \%$ in all physical activity values and a $28 \%$ increase in sedentary habits $(p<0.001)$. There was a widespread loss of muscle mass $(2.5 \% ; p<0.01)$ which could be interpreted as a temporary forced sarcopenia. As a conclusion, the importance of physical activity programs in this group is highlighted.
\end{abstract}

\section{Keywords}

Physical activity, Questionnarie GPAQ, sedentary habits, bioimpedance.
Carmen Gutiérrez-Cruz

$<$ cgutierrezcr.granada@hospitala rias.es>

Fundación Purísima Concepción de Granada. España

\section{Saray Muñoz-López}

<smunozl.granada@hospitalarias. es>

Fundación Purísima Concepción de Granada. España

\section{Andrés Román-Espinaco}

<aroman.granada@hospitalarias. es>

Fundación Purísima Concepción de Granada. España

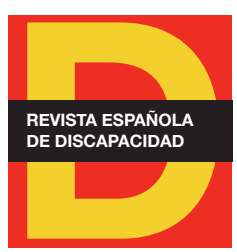

Para citar:

Gutiérrez-Cruz, C. et al. (2021). Efecto del confinamiento COVID-19 sobre la composición corporal en residentes con discapacidad intelectual. Revista Española de Discapacidad, 9(1), pp. 141-152.

Doi: $<$ https://doi.org/10.5569/23405104.09.01.07>

Fecha de recepción: 30-08-2020 Fecha de aceptación: 19-05-2021 


\section{Introducción ${ }^{1}$}

El confinamiento ha sido la medida extrema para el control de la pandemia debida a la COVID-19, una medida que ha supuesto cambios importantes en los hábitos de las personas. Las residencias para las personas con discapacidad intelectual o del desarrollo, han sido especialmente sensibles a estos cambios, lo que ha supuesto la restricción de movimientos, cancelación de actividades grupales, prohibición de visitas y cambios organizativos en los recursos y los profesionales que los asisten. Aunque los datos indican que las distintas discapacidades intelectuales no son factores de riesgo para la COVID-19, este colectivo tiene una prevalencia de enfermedades físicas de 2,5 veces más alta que la población general (Plena Inclusión, 2020), así como un mayor deterioro de su calidad de vida conforme avanzan en edad (Millán-Calenti et al., 2003), lo que les hace especialmente vulnerables a las medidas adoptadas.

La necesaria reestructuración de programación que se ha realizado en estas residencias ha obligado a restringir y/o eliminar los programas dedicados al desarrollo de actividades recreativas, lúdicas o deportivas que tienen como objetivo mejorar la salud y la calidad de vida de estas personas a través del ejercicio físico. Aunque el tiempo libre en las residencias es muy alto, la supresión de estos programas suele provocar un incremento considerable de horas dedicadas al ocio pasivo (Son et al., 2016; Baek, 2014) y con ello, la eliminación de una cierta adherencia al ejercicio físico que tanto habría costado alcanzar debido los bajos niveles motivacionales que tienen estas personas hacia la práctica del ejercicio físico, con respecto a la población general del mismo rango (Temple et al., 2006; Cuesta-Vargas et al., 2011). La consecuencia es una reducción de la actividad cardiorrespiratoria y muscular, además de un incremento en el riesgo de obesidad en los adultos con discapacidad intelectual leve o moderada (Bouzas et al., 2019), la cual está asociada a otras afecciones crónicas como la diabetes tipo Il o la hipertensión arterial, lo que convierte al sedentarismo en una amenaza importante para la salud de los usuarios de estos centros (Einarsson et al., 2015; Wyszyńska et al., 2020), especialmente cuando investigaciones precedentes han constatado que la obesidad es más frecuente entre las personas con discapacidad intelectual con respecto a la población general (Casey, 2013; Yamaki et al., 2005).

El confinamiento obligado debido a la COVID-19 ha supuesto para la mayoría de las residencias una situación temporal donde se han reducido drásticamente las actividades programadas que podrían estar asociadas a la actividad física, como los desplazamientos (visitas a centros comerciales y paseos por la ciudad), las actividades recreativas (marchas y juegos grupales), el deporte (natación, fútbol, baloncesto y otros), así como una reducción significativa de los programas grupales prescritos en el ámbito psicomotor (ejercicio terapéutico y rehabilitación). Sin embargo, al tratarse de centros residenciales, los usuarios han mantenido la misma dieta y el mismo tratamiento farmacológico, lo que nos proporciona una situación óptima para evaluar el efecto que ha podido tener la reducción de ejercicio físico sobre la composición corporal en los usuarios de residencias de personas con discapacidad intelectual.

A partir de los antecedentes expuestos, parece razonable considerar la importancia que tiene la evaluación y seguimiento de la composición corporal para el control de la salud y calidad de vida de los usuarios residentes con discapacidad intelectual. La complejidad y el alto coste de los sistemas de registro, así como la

1. Agradecimientos: a la Universidad de Granada y al Instituto Mixto de Deporte y Salud (IMUDS) por brindarnos la oportunidad de poder utilizar sus medios y sistemas. A la Fundación Purísima Concepción de Granada por su colaboración y en concreto a la residencia de RDP. 
capacitación exigida para su adquisición, han podido ser los motivos de su escasa implantación en estos centros. Sin embargo, el bajo coste actual, la confiabilidad y sencillez de los sistemas basados en la impedancia bioeléctrica (BIA) para la medida de la composición corporal (Havinga-Top et al. , 2015), ha permitido que ciertas residencias de poblaciones especiales hayan podido acceder a esta evaluación. Este ha sido el caso de la Residencia de Psicodeficientes de la Fundación Purísima Concepción de Granada donde, con la colaboración de la Universidad de Granada, suelen realizarse estas evaluaciones como medida de control de la salud y la calidad de vida de sus usuarios. Con este objetivo, una de esas evaluaciones se realizó escasos días antes de comenzar el confinamiento COVID-19, cuyas medidas restrictivas y programáticas se han utilizado como variable independiente para convertir lo que se había ideado como un servicio a los usuarios en un diseño de investigación que nos ha permitido evaluar su efecto sobre la composición corporal de nuestros usuarios.

Según lo expuesto, el propósito de este estudio ha sido doble: A) Describir como han afectado las medidas de confinamiento COVID-19 durante 17 semanas, sobre el grado de participación en las actividades cotidianas que implican esfuerzo físico y B) Comprobar el efecto que han tenido esos cambios sobre la composición corporal en los usuarios de la residencia de la Fundación Purísima Concepción de Granada. Teniendo en cuenta los antecedentes expuestos, consideramos como hipótesis que habrá existido una reducción muy significativa de aquellas actividades que implican esfuerzo físico, siendo sustituidas por ocio pasivo y que estos cambios han supuesto modificaciones en la composición corporal de los residentes, donde se incrementará la masa grasa, mientras que la masa muscular se verá reducida.

\section{Método}

\subsection{Aproximación experimental al problema}

Para probar la hipótesis se ha utilizado un diseño de medidas repetidas donde cada usuario fue evaluado antes y después del confinamiento COVID-19 (pre-confinamiento y post-confinamiento). Según los objetivos expuestos, la variable independiente ha sido el periodo de confinamiento COVID-19 producido en la residencia de la Fundación Purísima Concepción de Granada en aplicación de la Guía práctica emitida por el Ministerio de Sanidad, Consumo y Bienestar Social para residencias de personas con discapacidad intelectual durante la COVID-19, sin que hayan existido casos positivos de la COVID-19 durante dicho periodo de confinamiento. Esta estrategia de investigación nos ha permitido evaluar su efecto sobre los diferentes grados de actividad física, así como los cambios producidos en la composición corporal de los usuarios, registrándose para ello las siguientes variables dependientes: a) Masa total (peso); $b$ ) masa muscular esquelética, c) masa grasa; $d$ ) grado de obesidad abdominal (relación cintura-cadera); e) índice de masa corporal (IMC) y f) el metabolismo basal.

\subsection{Participantes}

Han participado 42 personas adultas, usuarias de la residencia de la Fundación Purísima Concepción de Granada, todas ellas con discapacidad intelectual en diferentes grados. En la tabla 1 se presentan las medi- 
das de tendencia central sobre características más significativas de los usuarios que fueron incluidos en el estudio. El grado de discapacidad se ha estimado a partir de los informes del equipo psicológico del centro y para la evaluación de los niveles de actividad física se han utilizado los criterios de la Organización Mundial de la Salud (OMS), donde se consideran como personas activas aquellas que acumulan más de 150 minutos semanales de actividad física moderada o 75 minutos de actividad física de intensidad vigorosa o una combinación de ambas superior a 225 minutos en bloques mínimo de 10 minutos continuos.

A partir del consentimiento de la Dirección y del coordinador de la residencia, los participantes firmaron el consentimiento informado en función de la voluntad de los padres, madres o tutores legales. La investigación cumplía con los requisitos éticos de la Declaración de Helsinki y su posterior revisión de 2013.

\begin{tabular}{l}
\hline Tabla 1. Características generales más significativas de los participantes \\
\begin{tabular}{|l|l|c|}
\hline \multicolumn{2}{|l|}{ Total de participantes $(N)$} & 42 \\
\hline \multirow{2}{*}{ Sexo } & Hombre & 25 \\
\cline { 2 - 3 } & Mujer & 17 \\
\hline \multirow{2}{*}{ Edad (años) } & Ligera & $35,7 \pm 11,2$ \\
\hline \multirow{2}{*}{$\begin{array}{l}\text { Grado de discapacidad } \\
\text { intelectual }\end{array}$} & Moderada & 15 \\
\cline { 2 - 3 } & Severa & 15 \\
\hline \multirow{2}{*}{ Nivel de actividad física } & Activos & 12 \\
\cline { 2 - 3 } & Sedentarios & 15 \\
\hline Talla $(m)$ & & 27 \\
\hline Masa $(\mathrm{kg})$ & & $1,64 \pm 0,11$ \\
\hline
\end{tabular}
\end{tabular}

Media \pm Desviación estándar

Fuente: elaboración propia.

\subsection{Material y sistemas de registro}

Para evaluar el grado de actividad física de los participantes se ha utilizado el Global Physical Activity Questionnaire (GPAQ), un cuestionario desarrollado por la Organización Mundial de la Salud (OMS) para evaluar diferentes niveles de actividad física y los comportamientos sedentarios en tres campos de actuación: a) en el trabajo (en este caso se han considerado todas aquellas actividades programadas y, por lo tanto, de obligado cumplimiento para ellos), b) al desplazarse de un sitio a otro, al menos 10 minutos consecutivos (al tratarse de personas internas residentes no se ha considerado este campo) y c) en el tiempo libre (se han considerado todas aquellas actividades físicas cotidianas que se realizan de forma voluntaria). Para los dos campos de actuación utilizados en este trabajo se han considerado dos niveles: i) actividad física intensa, cuando implican una aceleración importante de la respiración o del ritmo cardiaco, al menos 10 minutos consecutivos y ii) actividad física moderada, cuando supone una ligera aceleración de la respiración o del ritmo cardiaco, al menos 10 minutos consecutivos. 
Para la aplicación de dicho cuestionario se utilizó la entrevista a los participantes con la ayuda del equipo asistencial de la residencia. Los resultados del cuestionario se expresan a través de la Unidad Metabólica Equivalente (MET), una medida que se utiliza normalmente para analizar la actividad física. La guía del Cuestionario Mundial sobre Actividad Física (GPAQ) define un MET como $1 \mathrm{kcal} / \mathrm{kg} / \mathrm{hora}$ y equivale a la energía consumida por el cuerpo en reposo. Siguiendo dicha guía, en este trabajo se ha utilizado el MET-minutos/ semana como indicador del nivel de actividad física. Para su cálculo se han considerado los criterios de conversión estimados por la Organización Mundial de la Salud (OMS) para el análisis de los datos de este cuestionario, donde se estima que el consumo de calorías de una persona es cuatro veces más alto cuando está activo (actividades moderadas) y ocho veces más alto cuando está muy activo (actividades vigorosas), considerándose a una persona activa cuando la combinación entre ambas intensidades sea mayor a 600 MET- minutos en bloques mínimos de 10 minutos continuos.

Para el análisis de la composición corporal se ha utilizado el sistema de registro InBody-230 (Biospace, Seoul, Corea del Sur). Se trata de un sistema que utiliza la impedancia o resistencia que ofrece el cuerpo al paso de dos corrientes con frecuencias diferentes $(20 \mathrm{kHz}$ y $100 \mathrm{kHz}$ ). Para evitar errores sistemáticos en la medida de la masa corporal, su registro se realizó en la misma franja horaria para cada participante manteniendo el mismo vestuario deportivo. En ese sentido metodológico es necesario considerar que la precisión de estos datos depende de ecuaciones de regresión específicas para cada población, por lo que es necesario ser cauto cuando éstos se comparan con otras poblaciones (Casey, 2013; Sung et al., 2001). Sin embargo, al tratarse de un diseño de medidas repetidas, donde se evalúan los cambios que se producen en cada participante (intrasujeto), esta observación sobre la validez de los datos no debería suponer un problema.

\subsection{Procedimientos}

Las dos tomas de datos se realizaron en las instalaciones propias de la Fundación Purísima Concepción de Granada, la primera de ellas (pre-confinamiento) se realizó entre los días 3 y 5 de marzo de 2020 y la segunda después de 17 semanas de confinamiento (post-confinamiento), entre los días 5 y 7 de julio de 2020. Para cada toma de datos se necesitaron dos sesiones: en la primera se realizaron los cuestionarios GPAQ (Global Physical Activity Questionnaire), donde el participante estaba acompañado por la psicomotricista del centro. Aunque las preguntas se dirigían al participante, las respuestas siempre debían estar confirmadas por las dos personas del equipo asistencial.

Al día siguiente se registraron los datos de composición corporal. Después de quitarse cualquier adorno personal metálico, como relojes de pulsera o similares, calcetines y sudadera, los participantes fueron informados sobre el método de medición y la postura corporal que deberían adoptar para realizar una medición precisa. Cuando se constataba que los participantes habían entendido la información dada, se introducían los datos personales requeridos por el sistema (edad, sexo y talla) y se procedía a la limpieza de las plantas de los pies y las palmas de las manos, utilizando para ello toallitas húmedas. A continuación se les ayudó a mantener la postura adecuada en el lugar marcado en el dispositivo. Se realizaron cinco registros de forma consecutiva, seleccionándose para el análisis el registro cuyo valor de masa grasa era el mediano de los cinco. Las dos tomas de datos pre y post se realizaron el mismo día de la semana y a la misma hora para cada participante por lo que se mantuvo constante la actividad previa en las dos evaluaciones. 


\subsection{Análisis estadístico}

Los datos descriptivos son presentados como media \pm desviación estándar. El supuesto de distribución normal de las variables fue testado a través del test de Shapiro-Wilk. Ninguna de las variables relacionadas con la cantidad de actividad física realizada (expresadas en MET) o el comportamiento sedentario (Tiempo sentado/semana) mostraron una distribución normal (todas $p<0,001$ ), mientras que todas las variables relacionadas con la composición corporal sí siguieron una distribución normal (p osciló entre 0,055 y 0,665). Consecuentemente, para determinar el efecto que ha tenido el confinamiento a partir de los dos registros (pre-confinamiento y post-confinamiento) se ha aplicado la prueba de Wilcoxon para las variables que no siguieron una distribución normal (niveles de actividad física y comportamiento sedentario) y la prueba t de Student para muestras relacionadas en las variables que siguieron una distribución normal (todas las variables relacionadas con la composición corporal). Para todo ello se ha utilizado el paquete estadístico SPSS (IBM SPSS versión 22.0, Chicago, IL, USA).

\section{Resultados}

En la tabla 2 se presentan los datos numéricos correspondientes al cuestionario Global Physical Activity Questionnaire (GPAQ) para todos los participantes $(\mathrm{N}=42)$. En ella se expresan los datos de tendencia central y estadística inferencial correspondiente a los valores MET-minutos/semana, para los dos campos y niveles de intensidad en actividad física y el comportamiento sedentario (tiempo sentado/semana) en las dos situaciones experimentales (pre-confinamiento y post- confinamiento). La primera observación que desprenden los datos expuestos es la alta desviación típica existente en todos los valores MET, lo que indica la alta dispersión o variabilidad en actividad física que existe entre las personas con discapacidad intelectual. Los datos de tendencia central antes del confinamiento alcanzan valores próximos a los indicadores para personas activas $(559.8 \pm 574.0$, para MET Total). Sin embargo, estos mismos datos se reducen de forma significativa durante el confinamiento, alcanzando valores medios propios de personas muy sedentarias $(74.3 \pm 171.6$ para MET Total; $\mathrm{p}<0.001)$. El comportamiento sedentario general o tiempo medio que permanecen sentados los usuarios indica una alta tasa media de sedentarismo antes del confinamiento, la cual aumenta de forma muy significativa durante el confinamiento $(p<0.001)$.

En la tabla 3 se presentan estos mismos datos para las mujeres $(\mathrm{N}=17)$ y los hombres $(\mathrm{N}=25)$ que han participado en el estudio. La primera observación que se puede hacer es que todos los valores medios de MET-minutos/ semana son inferiores en las mujeres con respecto a los hombres y muy alejados de los valores mínimos para considerar a este grupo como personas activas (>600 MET-minutos/semana totales), todo lo contrario que ocurre para los hombres. Con respecto a los cambios producidos durante el confinamiento, en los dos sexos se mantiene una tendencia similar a la descrita para todos los participantes, reduciéndose de forma significativa todos los registros MET durante el confinamiento e incrementándose la media correspondiente al tiempo que permanecen sentados. El grupo de mujeres no ha registrado valores MET para los niveles donde se exige una actividad física vigorosa (MET-trabajo vigoroso y MET-tiempo libre moderado) en ninguna de las dos situaciones experimentales (pre-confinamiento y post-confinamiento) y la alta desviación típica de los datos registrados para MET-tiempo libre moderado ha provocado la no significación estadística entre sus medias. 
Tabla 2. Valores de actividad física expresados en Unidad Metabólica Equivalente (MET-minutos/semana) y comportamiento sedentario, expresado por el tiempo que permanecen sentados durante una semana (tiempo sentado/semana)

\begin{tabular}{|l|c|c|c|}
\hline Variable & Pre-confinamiento & Post-confinamiento & P \\
\hline MET Trabajo vigoroso & $21,2 \pm 55,6$ & $0 \pm 0$ & 0,026 \\
\hline MET Trabajo moderado & $274,1 \pm 328,4$ & $0 \pm 0$ & $<0,001$ \\
\hline MET Tiempo libre vigoroso & $30,9 \pm 86,0$ & $0 \pm 0$ & 0,039 \\
\hline MET Tiempo libre moderado & $230,3 \pm 289,6$ & $74,3 \pm 171,7$ & $<0,001$ \\
\hline MET Total & $559,8 \pm 574,0$ & $74,3 \pm 171,6$ & $<0,001$ \\
\hline Tiempo sentado/semana $(\min )$ & $283,3 \pm 60,3$ & $390,5 \pm 74,7$ & $<0,001$ \\
\hline
\end{tabular}

Fuente: elaboración propia.

\begin{tabular}{|c|c|c|c|}
\hline Variable & Pre-confinamiento & Post-confinamiento & $\mathbf{P}$ \\
\hline \multicolumn{4}{|c|}{ MUJERES } \\
\hline MET Trabajo vigoroso & $0 \pm 0$ & $0 \pm 0$ & - \\
\hline MET Trabajo moderado & $168,3 \pm 263,9$ & $0 \pm 0$ & 0,003 \\
\hline MET Tiempo libre vigoroso & $0 \pm 0$ & $0 \pm 0$ & - \\
\hline MET Tiempo libre moderado & $138,4 \pm 263,9$ & $42,4 \pm 126,9$ & 0,068 \\
\hline TOTAL (MET) & $315,1 \pm 362,3$ & $42,4 \pm 126,9$ & $<0,001$ \\
\hline Tiempo sentado (min) & $304,5 \pm 46,5$ & $403,5 \pm 6,9$ & 0,003 \\
\hline \multicolumn{4}{|c|}{ HOMBRES } \\
\hline MET trabajo vigoroso & $35,5 \pm 68,9$ & $0 \pm 0$ & 0,026 \\
\hline MET trabajo moderado & $346,1 \pm 378,7$ & $0 \pm 0$ & $<0,001$ \\
\hline MET tiempo libre vigoroso & $51,8 \pm 107,3$ & $0 \pm 0$ & 0,039 \\
\hline MET tiempo libre moderado & $292,8 \pm 294,6$ & $96,0 \pm 196,0$ & 0,001 \\
\hline TOTAL (MET) & $726,2 \pm 636,50$ & $96,0 \pm 196$ & $<0,001$ \\
\hline Tiempo sentado (min) & $271,2 \pm 65,1$ & $384,0 \pm 81,2$ & $<0,001$ \\
\hline
\end{tabular}

Fuente: elaboración propia.

En la tabla 4 se presenta la estadística descriptiva e inferencial de todos los participantes para las variables relacionadas a la composición corporal en las dos condiciones experimentales (pre-confinamiento y post-confinamiento). Los datos ponen de manifiesto que han existido claras diferencias entre las medias correspondientes a la masa total registrada (peso), antes y después del confinamiento ( $\mathrm{p}<.001)$, reduciéndose dicha masa una media de $1.85 \pm 1.67 \mathrm{~kg}$ después de las 17 semanas de confinamiento. Los datos expuestos también señalan una reducción significativa entre las medias de la masa muscular $(p<0.01)$, sin embargo, no han existido diferencias estadísticamente significativas entre las medias para la masa grasa. 
Los datos relativos al Índice de Masa Corporal (IMC) indican que han existido claras diferencias entre las medias de sus datos registradas antes y después del confinamiento $(p<0.001)$, reduciéndose una media del $2.5 \%$ los datos obtenidos después del confinamiento (post-confinamiento), con respecto a la registrada antes de iniciarse el confinamiento (pre-confinamiento). Finalmente, los datos sobre el metabolismo basal indican que existen ciertas diferencias estadísticamente significativas entre las medias $(p<0.05)$, reduciéndose los datos obtenidos una media del $1.7 \%$ después del confinamiento con respecto a los datos previos.

Tabla 4. Resultados estadísticos de las variables más significativas relacionadas con la composición corporal para
todos los participantes en las dos situaciones experimentales (pre-confinamiento y post-confinamiento)
\begin{tabular}{|l|c|c|c|}
\hline Variable & Pre-confinamiento & Post-confinamiento & t \\
\hline Masa total $(\mathrm{kg})$ & $73,89 \pm 11,43$ & $72,04 \pm 11,05$ & $7,17^{\star \star *}$ \\
\hline Masa muscular $(\mathrm{kg})$ & $27,00 \pm 5,76$ & $25,76 \pm 5,47$ & $3,21^{\text {** }}$ \\
\hline Masa grasa $(\mathrm{kg})$ & $24,82 \pm 10,39$ & $24,15 \pm 11,24$ & 0,91 \\
\hline Obesidad abdominal $(\mathrm{cm})$ & $0,94 \pm 0,10$ & $0,94 \pm 0,09$ & 0,152 \\
\hline IMC $\left(\mathrm{kgm}^{-2}\right)$ & $27,67 \pm 4,76$ & $26,99 \pm 4,63$ & $6,52^{\star * *}$ \\
\hline Metabolismo $(\mathrm{kcal})$ & $1430,02 \pm 205,64$ & $1405,40 \pm 193,43$ & $2,45^{\star}$ \\
\hline
\end{tabular}

${ }^{* \star *} p<0.001 ;{ }^{* \star} p<0.01 ;{ }^{*} p<0.05$

Fuente: elaboración propia.

En la tabla 5 se presentan estos mismos datos distinguiendo entre las mujeres y los hombres que han participado en el estudio. Los resultados de la estadística inferencial mantienen una tendencia similar en la mayoría de las variables presentadas en la tabla 4 para todos los participantes. Sólo han existido ciertas diferencias estadísticas entre las medias del metabolismo basal, reduciéndose de forma significativa en los hombres después del confinamiento $(p<0.05)$, mientras no han existido diferencias estadísticamente significativas en el grupo de mujeres. Los datos también indican que la diferencia entre las medias para la masa grasa ha sido mayor en el grupo de las mujeres con respecto a los hombres, aunque sin alcanzar en ninguno de los dos grupos niveles de significación estadística. En este sentido, mientras las mujeres han reducido su masa grasa una media de $4.62 \%$, los hombres sólo lo han hecho en el $0.9 \%$, aunque también habría que tener en cuenta que las mujeres poseen valores medios de masa grasa muy superiores a los hombres.

Tabla 5. Resultados estadísticos de las variables más significativas relacionadas con la composición corporal para hombres y mujeres en las dos situaciones experimentales (pre-confinamiento y post-confinamiento)

\begin{tabular}{|l|c|c|c|}
\hline Variable & Pre-confinamiento & Post-confinamiento & t \\
\hline \multicolumn{4}{|c|}{ MUJERES } \\
\hline Masa total $(\mathrm{kg})$ & $69,96 \pm 11,34$ & $68,48 \pm 11,37$ & $3,85^{\star \star}$ \\
\hline Masa muscular $(\mathrm{kg})$ & $21,48 \pm 2,81$ & $20,92 \pm 2,64$ & $2,98^{\star \star}$ \\
\hline Masa grasa $(\mathrm{kg})$ & $30,07 \pm 10,63$ & $28,68 \pm 10,85$ & 1,96 \\
\hline Obesidad abdominal $(\mathrm{cm})$ & $0,99 \pm 0,13$ & $0,98 \pm 0,11$ & 0,73 \\
\hline IMC $\left(\mathrm{kgm}^{-2}\right)$ & $29,75 \pm 5,35$ & $29,04 \pm 5,29$ & $4,05^{\star \star}$ \\
\hline Metabolismo $(\mathrm{kcal})$ & $1236,18 \pm 110,48$ & $1223,65 \pm 100,29$ & 1,47 \\
\hline
\end{tabular}




\begin{tabular}{|c|c|c|c|}
\hline Variable & Pre-confinamiento & Post-confinamiento & $\mathbf{t}$ \\
\hline \multicolumn{4}{|c|}{ HOMBRES } \\
\hline Masa total $(\mathrm{kg})$ & $76,57 \pm 10,91$ & $74.46 \pm 10,37$ & $6,13^{\star \star \star}$ \\
\hline Masa muscular $(\mathrm{kg})$ & $30,79 \pm 3,94$ & $29.04 \pm 4,29$ & $2,72^{\star \star}$ \\
\hline Masa grasa $(\mathrm{kg})$ & $21,25 \pm 8,75$ & $21.06 \pm 10,63$ & 0,16 \\
\hline Obesidad abdominal $(\mathrm{cm})$ & $0,91 \pm 0,05$ & $0.91 \pm 0,06$ & $-0,72$ \\
\hline IMC $\left(\mathrm{kgm}^{-2}\right)$ & $26,57 \pm 3,81$ & $25,59 \pm 3,60$ & $5,03^{\star \star \star}$ \\
\hline Metabolismo (kcal) & $1561,84 \pm 139,24$ & $1529,00 \pm 133,78$ & $2,08^{*}$ \\
\hline
\end{tabular}

${ }^{* *} \mathrm{p}<0.001 ;{ }^{* *} \mathrm{p}<0.01 ;{ }^{*} \mathrm{p}<0.05$

Fuente: elaboración propia.

\section{Discusión}

Existe suficiente evidencia científica para afirmar que las personas con discapacidad intelectual son más sedentarias y alcanzan menos niveles de actividad física que las personas sin discapacidad (Temple et al., 2006; Boonman et al., 2019; Oviedo et al., 2019), siendo las mujeres más sedentarias que los hombres (Westrop et al., 2019), lo que se confirma con los datos expuestos en la tabla 2 y 3 . Los valores medios de MET-minutos-semana que alcanzan antes de iniciarse el confinamiento (559.8 \pm 574.0$)$ confirman esta evidencia, al no alcanzar el nivel mínimo para considerar que se trata de personas activas según criterios de la Organización Mundial de la Salud (OMS) (>600 MET-minutos/semana), siendo las mujeres más sedentarias que los hombres (315.1 \pm 362.3 y $726.2 \pm 636.50$, respectivamente). Sin embargo, los datos evidencian una alta variabilidad entre los usuarios de la residencia, posiblemente asociada a factores como la edad o el tipo y grado de discapacidad.

Los datos también indican una clara reducción de la actividad física y un incremento de los comportamientos sedentarios durante el confinamiento, con respecto a la registrada antes de iniciarse éste (ver tabla 3). Atendiendo a los valores de MET-minutos-semana totales, dicha reducción ha alcanzado valores medios del $89 \%(p<0.001)$, mientras que el tiempo que permanecen sentados se ha incrementado un $28 \%(p<0.001)$. Considerando que los usuarios mantuvieron la misma dieta y tratamiento farmacológico durante el confinamiento, los datos indican que se ha producido una reducción clara de actividad física y un incremento asociado de los hábitos sedentarios. En consecuencia, se constata que los posibles cambios en la composición corporal de los usuarios estarían provocados por la obligada inactividad física debido a las medidas para el control de la COVID-19 (en este trabajo no se han considerado los aspectos psicológicos y sociológicos derivados del confinamiento).

La primera observación que se puede hacer con respecto a los cambios que se han producido durante el confinamiento sobre la composición muscular es la pérdida de un 2,5\% del peso corporal y, como consecuencia de ello, también de IMC ( $p<0.001$-ver tabla 4 y 5), lo que parece sorprendente cuando se había reducido la actividad física de forma muy significativa y la dieta establecida por el centro se mantuvo estable. Sin embargo, es conveniente indicar que, al suprimir las salidas programadas durante los fines de semana 
(habitualmente una salida a la semana), también se eliminaron los suplementos que habitualmente ingerían durante estas actividades, lo que podría explicar una parte de la reducción de peso durante el confinamiento, pero no toda la pérdida registrada. Efectivamente, los datos indican que la disminución registrada en el peso corporal se ha debido a la pérdida de masa muscular y no tanto a la reducción de la masa grasa (2.2\% y $0.3 \%$ para la masa muscular y masa grasa, respectivamente), constatándose diferencias significativas entre las medias de la masa muscular antes y después del confinamiento $(p<0.01)$, mientras que para la masa grasa no han existido diferencias estadísticamente significativas.

La escasa diferencia entre las medias del grado de obesidad abdominal (ver tablas 4 y 5), indica que la pérdida de peso no se ha focalizado en el cinturón pélvico, sugiriendo así una pérdida generalizada de masa muscular que es coherente con la ausencia de actividad física durante el confinamiento, lo que se podría interpretar como una sarcopenia debida a la inactividad física, la cual se vería incrementada en las mujeres al tener una menor masa muscular que los hombres antes de iniciarse el confinamiento (Hughes et al., 2002; Kalyani et al., 2014).

Teniendo en cuenta los bajos niveles de adherencia al ejercicio físico que tienen las personas con discapacidad intelectual (Temple et al., 2006; Cuesta-Vargas et al., 2011) y el hecho de permanecer en centros de atención residencial, su condición física sólo puede ser dependiente de los programas de actuación que se hayan podido diseñar con dicha finalidad. A partir de los datos expuestos en esta investigación es posible afirmar que la supresión temporal de estos programas conduce a un deterioro de la salud y calidad de vida de estas personas, lo que es coincidente con las aportaciones de otros investigadores (Son et al., 2016; Ayaso-Maneiro et al., 2013; Baek, 2014) para residentes de larga duración.

\section{Conclusiones y aplicación práctica}

La restricción de movimientos y la cancelación las actividades profesionales, lúdicas y recreativas debidas a las medidas tomadas para el control de la COVID-19, ha supuesto una reducción muy significativa de la actividad física y un incremento de actitudes sedentarias en personas residentes con discapacidad intelectual.

La reducción de actividad física durante las 17 semanas de confinamiento ha provocado una pérdida generalizada de masa muscular que podría interpretarse como una sarcopenia forzada debida a la inactividad física temporal. Un efecto que, de mantenerse en el tiempo y combinado con el deterioro por la edad, podría afectar gravemente a la salud y la calidad de vida de las personas con discapacidad intelectual que reciben atención permanente en las residencias.

Los resultados de esta investigación confirman las aportaciones de otros investigadores cuando ponen de manifiesto la importancia que tiene aplicar programas de actuación que implican actividad física y supongan una cierta adherencia al ejercicio físico de las personas residentes con discapacidad intelectual. 
Ayaso-Maneiro, J. et al. (2014). Aplicación de un programa de ejercicio terapéutico en población adulta con discapacidad intelectual. Apunts Sport Medicine, 49(182), pp. 45-52. https://doi.org/10.1016/j.apunts.2013.12.003.

Baek, S. H. (2014). An effect on mentally disabled persons' adaptation to behavior and satisfaction of sports activities. Journal Social Welfare and Disabiliy, 5, pp. 25-49.

Boonman, A. et al. (2019). Cardiopulmonary profile of individuals with intellectual disability. Medicine \& science in sport \& exercise, 51(9), pp. 1802-1808. https://doi.org/10.1249/MSS.0000000000001995.

Bouzas, S. et al. (2019). Effects of exercise on the physical fitness level of adults with intellectual disability: a systematic review. Disability and Rehabilitation, 41(26), pp. 3118-3140. https://doi.org/10.1080/09638288.2018.1 491646.

Casey, A. F. (2013). Measuring body composition in individuals with intellectual disability: A scoping review. Journal of Obesity, 2013, pp. 1-6. https://doi.org/10.1155/2013/628428.

Cuesta-Vargas A. L. et al. (2011). Physical fitness profile in adults with intellectual disabilities: Differences between levels of sport practice. Research Developmental Disabilities, 32, pp. 788-794. https://doi.org/10.1016/j. ridd.2010.10.023.

Einarsson, I. O. et al. (2015). Differences in physical activity among youth with and without intellectual disability. Medicine and Science in Sports and Exercise, 47(2), pp. 411-418. https://doi.org/10.1249/MSS.0000000000000412.

Havinga-Top, A. M. et al. (2015). Feasibility of bioelectrical impedance analysis in persons with severe intellectual and visual disabilities. Research in Developmental Disabilities, 47, pp. 126-134. https://doi.org/10.1016/j. ridd.2015.09.003.

Hughes V. A. et al. (2002). Longitudinal changes in body composition in older men and women: role of body weight change and physical activity. American Journal Clinical Nutrition, 76, pp. 473-481. https://doi.org/10.1093/ ajcn/76.2.473.

Kalyani, R. R. et al. (2014). Age-related and disease-related mucle loss: The effect of diabetes, obesity and other diseases. Diabetes \& Endocrinology, 2(19), pp. 819-829. https://doi.org/10.1016/S2213-8587(14)70034-8.

Millán-Calenti, J. C. et al. (2003). Intellectual disability and ageing: a new challenge for the twenty-first century. Revista Española de Geriatría y Gerontología, 38(5), pp. 266-274.

Oviedo G. R. et al. (2019). Physical activity and sedentary time in active and non-active adults with intellectual disability: A comparative study. International journal of environmental research and public health, 16(10), p. 1761. https://doi.org/10.3390/ijerph16101761.

Plena Inclusión (2020). Guía para residencias durante el COVID-19. Ministerio de Sanidad, Consumo y Bienestar Social. https://www.plenainclusion.org/wp-content/uploads/2021/03/plena_inclusion._guia_para_residencias_durante_el_covid19.pdf.

Son, S. et al. (2016). Effects of a walking exercise program for obese individuals with intellectual disability staying in a residential care facility. Journal Physical Theraphy Sciences, 28, pp. 788-793. https://doi.org/10.1589/ jpts.28.788. 
Sung, R. Y. T. et al. (2001). Measurement of body fat using legtoleg impedance. Archives of Disease in Childhood, 85, pp. 263-267. https://doi.org/10.1155/2013/628428.

Temple, V. A. et al. (2006). Physical activity of adults with mental retardation: review and research needs. American Journal of Health Promotion, 21, pp. 2-12. https://www.doi.org/10.1177/089011710602100103.

Westrop. S. C. et al. (2019). Gender differences in physical activity and sedentary behavior in adults with intellectual disabilities: A systematic review and meta-analysis. Journal of applied research in intellectual disabilities, 32, pp. 1359-1374. https://doi.org/10.1111/jar.12648.

Wyszyńska, J. et al. (2020). Associations between adiposity indicators and hypertension among children and adolescents with intellectual disability-A case-control study. Journal of Applied Research in Intellectual Disabilities. En prensa.

Yamaki, K. et al. (2005). Body weight status among adults with intelectual disability in the community. Mental Retardation, 43, pp. 1-10. https://doi.org/10.1352/0047-6765(2005)43<1:BWSAAW>2.0.CO;2. 